\title{
Identification of Transmembrane Protein in Prostate Cancer by the Escherichia coli Ampicillin Secretion Trap: Expression of CDON Is Involved in Tumor Cell Growth and Invasion
}

\author{
Tetsutaro Hayashi ${ }^{a}$ b Naohide Oue ${ }^{a}$ Naoya Sakamoto ${ }^{a}$ Katsuhiro Anami ${ }^{a}$ \\ Htoo Zarni Oo ${ }^{a}$ Kazuhiro Sentani ${ }^{a}$ Shinya Oharab Jun Teishimab

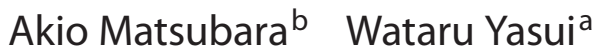

Departments of a Molecular Pathology and ${ }^{b}$ Urology, Hiroshima University Graduate School of Biomedical

Sciences, Hiroshima, Japan

\section{Key Words}

Prostate cancer • Escherichia coli ampicillin secretion trap • CDON

\begin{abstract}
Aims: Prostate cancer ( $\mathrm{PCa}$ ) is one of the most common malignancies worldwide. Genes expressed only in cancer tissue, and especially related to proteins located on the cell membrane, will be useful molecular markers for diagnosis and may also be good therapeutic targets. The aim of this study was to identify genes that encode transmembrane proteins present in PCa. Methods and Results: We generated Escherichia coli ampicillin secretion trap (CAST) libraries from $2 \mathrm{PCa}$ cell lines and normal prostate tissues. By sequencing 3,264 colonies from CAST libraries, we identified 18 candidate genes that encode transmembrane proteins present in $\mathrm{PCa}$. Quantitative RT-PCR analysis of these candidates revealed that STEAP1, ADAM9 and CDON were expressed much more highly in PCa than in 15 kinds of normal tissues. Among the candidates, CDON encodes the CDO protein, which is an orphan cell surface receptor of the immunoglobulin superfam-
\end{abstract}

ily. Additional quantitative RT-PCR revealed that $83 \%$ of $\mathrm{PCa}$ tissues showed CDON overexpression. Knockdown of CDON in DU145 cells induced 5-fluorouracil-induced apoptosis and inhibited invasion ability. Conclusion: These results suggest that CDON has a high potential as a therapeutic target for PCa.

Copyright $\odot 2011$ S. Karger AG, Basel

\section{Introduction}

Internationally, prostate cancer $(\mathrm{PCa})$ is the second most common cancer diagnosed among men, and is the sixth most common cause of cancer death among men [1]. Currently, the standard diagnostic marker for PCa is prostate-specific antigen (PSA) and the rapid incorporation of aggressive PSA testing has resulted in dramatically earlier identification of PCa [2]. Mortality from PCa is decreasing, which might be attributed to several factors, including increases in PSA screening and surgery, use of higher doses of radiotherapy, and earlier use of hormone therapy. However, the prognosis of advanced PCa

\section{KARGER}

Fax +4161306 1234 E-Mail karger@karger.ch www.karger.com
(C) 2011 S. Karger AG, Basel

$1015-2008 / 11 / 0785-0277 \$ 38.00 / 0$

Accessible online at:

www.karger.com/pat
Wataru Yasui, $\mathrm{MD}, \mathrm{PhD}$

Department of Molecular Pathology

Hiroshima University Graduate School of Biomedical Sciences

1-2-3 Kasumi, Minami-ku, Hiroshima 734-8551 (Japan)

Tel. +81 82257 5147, E-Mail wyasui@ hiroshima-u.ac.jp 
still remains unsatisfactory [3]. Therefore, identification of new therapeutic targets for PCa is important.

PCa develops as a result of multiple genetic and epigenetic alterations [4]. Better knowledge of changes in gene expression that occur during carcinogenesis may lead to improvements in diagnosis, treatment and prevention. Genes encoding transmembrane/secretory proteins expressed specifically in cancers may be ideal biomarkers for cancer diagnosis. If the function of the gene product is involved in the neoplastic process, this gene may constitute a therapeutic target [5].

In the present study, to identify genes that encode transmembrane proteins present in $\mathrm{PCa}$, we generated Escherichia coli ampicillin secretion trap (CAST) libraries from 2 PCa cell lines, LNCaP and DU145. CAST is a signal sequence trap method, developed by Ferguson et al. [6]. Signal peptides target secreted and transmembrane proteins to their appropriate subcellular location, and typically consist of $4-15$ hydrophobic amino acids that are flanked by a basic $\mathrm{NH} 2$ terminus and a polar $\mathrm{COOH}$ terminus [7]. A consensus sequence for the signal peptide has not been identified and, thus, standard molecular techniques are not well suited to identify such proteins. CAST is a survival-based signal sequence trap method that exploits the ability of mammalian signal sequences to confer ampicillin resistance to a mutant $\beta$ lactamase lacking the endogenous signal sequence [8]. We previously performed CAST analysis on 2 gastric cancer cell lines and identified several genes that encode transmembrane proteins present in gastric cancer [9]. However, CAST analysis of PCa has not been performed.

We report here the identification of genes that encode transmembrane proteins expressed in PCa. Among these, we focused on the CDON gene because this gene is frequently overexpressed in PCa, while its expression is narrowly restricted in normal tissues. $C D O N$, which encodes CDO (cell adhesion molecule-related/downregulated by oncogenes) protein, was cloned in 1997 from a cDNA library constructed from a rat embryo fibroblast cell line [10]. CDO protein is an orphan cell surface receptor of the immunoglobulin superfamily that is expressed on muscle precursor cells and developing muscles during mouse embryogenesis [10]. It positively regulates differentiation of myoblast cells [11], and is thought to be a strong candidate to mediate some of the effects of cell-cell contact that are important in myogenesis. However, the expression and function of CDON in human cancers including PCa have not been reported. In the present study, the effect of $C D O N$ knockdown was also investigated.

\section{Materials and Methods}

\section{Cell Lines}

LNCaP and DU145 PCa cell lines were purchased from American Type Culture Collection (Manassas, Va., USA). All cell lines were maintained in RPMI 1640 (Nissui Pharmaceutical, Tokyo, Japan) containing $10 \%$ fetal bovine serum (Whittaker, Walkersville, Md., USA) in a humidified atmosphere of $5 \% \mathrm{CO}_{2}$ and $95 \%$ air at $37^{\circ} \mathrm{C}$.

\section{CAST Library Construction}

pCAST was designed to contain the kanamycin resistance gene and the $\beta$-lactamase gene lacking the first 69 nucleotides encoding the endogenous signal peptide. EcoRI and BamHI sites were placed upstream of the mutant $\beta$-lactamase gene for directional cloning. CAST library construction was performed as described previously [6]. In brief, CAST cDNA libraries were generated from $2 \mu \mathrm{g}$ of mRNA with a random primer containing a BamHI restriction site for reverse transcription (SuperScript Choice System; Invitrogen, Carlsbad, Calif., USA). The EcoRIadapted cDNA was digested with BamHI, size fractionated, ligated into pCAST and plated onto Luris-Bertani/ampicillin. Individual colonies were picked and grown in $1.0 \mathrm{ml}$ Luris-Bertani with kanamycin in a 96-well format. Plasmid DNA was sequenced in 96-well format using a primer located within the $\beta$-lactamase gene. The pCAST vector was kindly provided by Prof. Jonathan M. Graff (Department of Developmental Biology, University of Texas Southwestern Medical Center, Dallas, Tex., USA).

\section{Tissue Samples}

Subjects were 15 patients with PCa who were referred to the Department of Urology, Hiroshima University Hospital, Hiroshima, Japan. All PCa samples were obtained by radical prostatectomy and confirmed to be node negative by pathological examination. None of 15 patients with PCa received preoperative treatment. Samples were frozen immediately in liquid nitrogen and stored at $-80^{\circ} \mathrm{C}$ until use. It was confirmed microscopically that the tumor specimens consisted mainly $(>50 \%)$ of cancer cells. Tumor staging was according to the TNM classification system. Because written informed consent was not obtained, identifying information for all samples was removed before analysis in order to provide strict privacy protection. This procedure was in accordance with the Ethical Guidelines for Human Genome/Gene Research of the Japanese Government. Noncancerous samples of heart, lung, stomach, small intestine, colon, liver, pancreas, kidney, bone marrow, peripheral leukocytes, spleen, skeletal muscle, brain, spinal cord and prostate were purchased from Clontech (Palo Alto, Calif., USA).

\section{Quantitative RT-PCR Analysis}

Total RNA was extracted with an RNeasy Mini Kit (Qiagen, Valencia, Calif., USA), and $1 \mu \mathrm{g}$ of total RNA was converted to cDNA with a first-strand cDNA synthesis kit (Amersham Biosciences Corp., Piscataway, N.J., USA). Quantitation of CDON mRNA levels was done by real-time fluorescence detection as described previously [12]. The CDON primer sequences were $5^{\prime}$ TGG AAA TGA AGC CCC TCA GT-3' and 5'-GAC GCT CTC CTC CGG CA-3'. PCR was performed with a SYBR Green PCR Core Reagents Kit (Applied Biosystems, Foster City, Calif., USA). Real-time detection of the emission intensity of SYBR green 
Table 1. Properties of sequenced ampicillin-resistant colonies

\begin{tabular}{|c|c|c|c|}
\hline & LNCaP & DU145 & $\begin{array}{l}\text { Normal } \\
\text { prostate }\end{array}$ \\
\hline Sequenced clones & 1,344 & 960 & 960 \\
\hline Human named genes & 234 & 228 & 224 \\
\hline Genes cloned in-frame upstream of & & & 72 \\
\hline the leaderless lactamase gene & 96 & 120 & \\
\hline Genes encoding secreted protein & 6 & 8 & 7 \\
\hline Genes encoding transmembrane protein & 39 & 55 & 37 \\
\hline
\end{tabular}

bound to double-stranded DNA was performed with an ABI PRISM 7700 sequence detection system (Applied Biosystems) as described previously [13]. ACTB-specific PCR products were amplified from the same RNA samples and served as an internal control.

\section{Evaluation of the Specificity of Gene Expression}

To evaluate the specificity of expression of each gene, a specificity index was calculated as follows: first, we identified the normal tissue in which the target gene expression was highest among the 15 normal tissues analyzed by quantitative RT-PCR (the mRNA expression level in this tissue was denoted as A). We then identified PCa among the 9 PCa samples in which the target gene expression was highest by quantitative RT-PCR (the mRNA expression level in this tissue was denoted as B). The ratio $\mathrm{B}$ to $\mathrm{A}$ was defined as the specificity index. When the specificity index of the target gene was $\geq 10$, the gene was considered to show a high specificity for PCa. When the specificity index of the target gene was $<10$ and $\geq 2$, the gene was considered to show a low specificity for PCa. When the specificity index of the target gene was $<2$, the gene was considered to show no specificity for PCa.

\section{RNA Interference}

To knockdown the endogenous CDON, RNA interference was performed. Small interfering RNA (siRNA) oligonucleotides for $\mathrm{CDON}$ and a negative control were purchased from Invitrogen. Three independent oligonucleotides were used for CDON siRNA. The CDON siRNA1 sequence was 5'-UAU GGA GAG AGC UUG CAC CAG CUU G-3'. The CDON siRNA2 sequence was 5'-AAC AAC GGG AUA CUU AGG GAU GCC C-3'. The CDON siRNA3 sequence was 5'-UUA UGC AGC CAU GAG AUA CGA GUG G-3'. Transfection was performed using Lipofectamine RNAiMAX (Invitrogen) according to the manufacturer's protocol. Briefly, 60 pmol siRNA and $10 \mu \mathrm{l}$ Lipofectamine RNAiMAX were mixed in $1 \mathrm{ml}$ RPMI medium (10 nmol/l final siRNA concentration). After 20 min of incubation, the mixture was added to the cells and these were plated on dishes for each assay. Forty-eight hours after transfection, cells were analyzed for all experiments.

\section{Western Blot Analysis}

For Western blot analysis, cells were lysed as described previously [14]. The lysates ( $40 \mu \mathrm{g})$ were solubilized in Laemmli sample buffer by boiling and then subjected to 10\% SDS-polyacrylamide gel electrophoresis followed by electrotransfer onto a nitrocellulose filter. The filter was incubated with the primary antibody against CDO protein (rabbit polyclonal, dilution 1:500; Sigma Chemical, St. Louis, Mo., USA). Peroxidase-conjugated anti-rabbit IgG was used in the secondary reaction. Immunocomplexes were visualized with an ECL Western Blot Detection System (Amersham Biosciences). $\beta$-Actin antibody (Sigma Chemical) was also used as a loading control.

Cell Growth, Apoptosis and in vitro Invasion Assays

The cells were seeded at a density of 1,000 cells per well in 96-well plates. Cell growth was monitored after 1 and 2 days by 3-(4,5-dimethylthiazol-2-yl)-2,5-diphenyltetrazolium bromide (MTT) assay [15]. For apoptosis assay, cultured cells were treated for $48 \mathrm{~h}$ with $2 \mathrm{mM} 5$-fluorouracil (5-FU), and apoptosis was evaluated with an APOPercentage (Biocolor Ltd., Belfast, Ireland) in vitro apoptosis assay kit, according to the manufacturer's instructions. Modified Boyden chamber assays were performed to examine invasiveness as described previously [16]. Cells were plated at 10,000 cells per well in RPMI 1640 medium plus $1 \%$ serum in the upper chamber of a Transwell insert ( $8 \mu \mathrm{m}$ pore diameter; Chemicon, Temecula, Calif., USA) coated with Matrigel. Medium containing $10 \%$ serum was added in the bottom chamber. After 1 and 2 days, cells in the upper chamber were removed by scraping and the cells remaining on the lower surface of the insert were stained with CyQuant GR dye to assess the number of cells.

\section{Results}

\section{Generation of CAST Libraries}

To identify genes that encode transmembrane proteins present in $\mathrm{PCa}$, we generated CAST libraries from $2 \mathrm{PCa}$ cell lines (LNCaP and DU145) and normal prostate tissues. We sequenced 1,344, 960 and 960 ampicillin-resistant colonies from each CAST library. We compared these sequences to those deposited in the public databases using BLAST (accessed at http://blast.ncbi.nlm.nih. gov/Blast.cgi), and evaluated the subcellular localization of the gene products using data from GeneCards (accessed at http://www.genecards.org/index.shtml). The properties of sequenced ampicillin-resistant colonies are shown in table 1 . In total, we found that 39,55 and 37 
genes encoded transmembrane proteins, and 6, 8 and 7 genes encoded secreted proteins from LNCaP, DU145 and normal prostate CAST libraries, respectively. Because the purpose of this study was to identify genes that encode transmembrane proteins present in $\mathrm{PCa}$, we focused on transmembrane proteins.

\section{Identification of Genes Expressed More Highly in PCa} than in Normal Tissues

To identify genes expressed specifically in PCa, we compared the gene list from each PCa cell line CAST library to the normal prostate CAST library. We only selected genes that were detected at least twice in each PCa cell line CAST library. In addition, genes were selected that were not found in the normal prostate CAST library. We obtained 6 candidates from LNCaP and 14 candidates from DU145. In total, 18 individual candidate genes were identified (table 2). To confirm that these candidates were PCa specific, quantitative RT-PCR was performed to measure the expression of these candidates in $9 \mathrm{PCa}$ and 15 normal tissue samples. Representative results are shown in figure 1. Expression of the 18 candidate genes was not necessarily specific for PCa. However, several genes showed much higher expression in PCa than in normal tissues. We then focused on cancer specificity by calculating the specificity index for each gene (table 2). Of the 18 candidates, STEAP1 was found to show a high specificity for PCa, and 2 genes, $A D A M 9$ and $C D O N$, were found to show a low specificity for PCa. The expression levels of the 18 genes in PCa tissue samples were also compared to those in normal prostate tissue samples. As shown in table 2, overexpression (tumor/normal prostate tissue ratio $>2$ ) was observed for all 18 genes. Among the 9 PCa cases, corresponding nonneoplastic prostate tissue samples were available for quantitative RT-PCR from 5 cases. The expression levels of the 18 genes in 5 samples of corresponding nonneoplastic prostate tissue were analyzed by quantitative RT-PCR. Frequent overexpression (tumor/nonneoplastic prostate tissue ratio $>2$ ) was found for all 18 genes (data not shown).

Overexpression of STEAP1 and ADAM9 in PCa has been reported $[17,18]$; however, expression of $C D O N$ in human cancers including PCa has not been investigated. Therefore, expression of $C D O N$ was analyzed by quantitative RT-PCR in an additional 6 PCa samples and corresponding nonneoplastic prostate samples. We calculated the ratio of $C D O N$ mRNA expression levels between PCa tissue ( $\mathrm{T}$ ) and corresponding nonneoplastic prostate (N). T-to-N ratios $>2$ were considered to represent overexpression. Among 6 cases, 5 (83\%) cases showed CDON overexpression.
Table 2. Summary of quantitative RT-PCR analysis of candidate genes specifically expressed in $\mathrm{PCa}$

\begin{tabular}{|c|c|c|c|c|c|}
\hline \multirow[t]{2}{*}{ Gene name } & \multicolumn{2}{|c|}{$\begin{array}{l}\text { Normal organ with } \\
\text { highest expression }\end{array}$} & \multirow[t]{2}{*}{$\begin{array}{l}\mathrm{PCa} \\
(\mathrm{B})^{1}\end{array}$} & \multirow{2}{*}{$\begin{array}{l}\text { Specificity } \\
\text { index } \\
\text { (B/A) }\end{array}$} & \multirow{2}{*}{$\begin{array}{l}\mathrm{PCa} \\
\text { case } \\
\text { No. }^{2}\end{array}$} \\
\hline & organ & $\begin{array}{l}\text { mRNA } \\
\text { expression } \\
\text { level (A) }\end{array}$ & & & \\
\hline
\end{tabular}

\begin{tabular}{|c|c|c|c|c|c|}
\hline \multicolumn{6}{|c|}{ High specificity } \\
\hline STEAP1 & pancreas & 7.51 & $93.5^{\dagger}$ & 12.5 & 9 \\
\hline \multicolumn{6}{|c|}{ Low specificity } \\
\hline$A D A M 9$ & prostate & 1.0 & 6.8 & 6.8 & 5 \\
\hline$C D O N$ & skeletal muscle & 11.1 & 24.9 & 2.2 & 9 \\
\hline \multicolumn{6}{|l|}{ No specificity } \\
\hline CTCL & pancreas & 4.2 & 8.1 & 1.9 & 7 \\
\hline DSC2 & heart & 4.8 & 6.7 & 1.4 & 8 \\
\hline TNFRSF10D & pancreas & 30.7 & 31.1 & 1.0 & 4 \\
\hline DNAJB14 & heart & 4.3 & 3.7 & 0.9 & 4 \\
\hline$D L G 1$ & pancreas & 7.7 & 6.1 & 0.8 & 3 \\
\hline$A D A M 17$ & pancreas & 3.8 & 2.8 & 0.7 & 2 \\
\hline SLC30A9 & heart & 32.0 & 20.2 & 0.6 & 5 \\
\hline PTP4A1 & skeletal muscle & 30.0 & 18.2 & 0.6 & 5 \\
\hline$S G C E$ & heart & 17.2 & 9.3 & 0.5 & 2 \\
\hline TMEM161B & pancreas & 11.2 & 5.9 & 0.5 & 4 \\
\hline STX4 & heart & 12.2 & 4.3 & 0.4 & 4 \\
\hline LYSMD3 & skeletal muscle & 9.3 & 3.1 & 0.3 & 1 \\
\hline TM9SF3 & pancreas & 78.8 & 23.4 & 0.3 & 4 \\
\hline ITGB1 & heart & 23.4 & 6.7 & 0.3 & 2 \\
\hline TFRC & heart & 42.5 & 10.4 & 0.2 & 4 \\
\hline
\end{tabular}

The units are arbitrary. Target mRNA expression levels were standardized to $1.0 \mu \mathrm{g}$ total RNA from normal prostate as 1.0.

${ }^{1}$ With highest expression mRNA expression level.

${ }^{2}$ With mRNA expression level 2-fold greater than normal prostate. ${ }^{\dagger}$ Arbitrary.

\section{Effect of CDON Inhibition on Cell Growth and}

Invasive Activity

$\mathrm{CDO}$ protein is thought to be a strong candidate to mediate some of the effects of cell-cell contact that are important in myogenesis; however, the function of CDON in human cancers including $\mathrm{PCa}$ has not been reported. We performed Western blot analysis in LNCaP and DU145 cell lines. High CDO protein expression was found in DU145 cells as a band of approximately 139 $\mathrm{kDa}$, and $\mathrm{LNCaP}$ cells had low or no CDO protein expression (fig. 2a). In CAST analysis, colonies containing CDON gene were detected twice in the DU145 CAST library, whereas no colonies with this gene were detected in the LNCaP CAST library, indicating that Western blot analysis of CDO protein was consistent with CAST anal- 


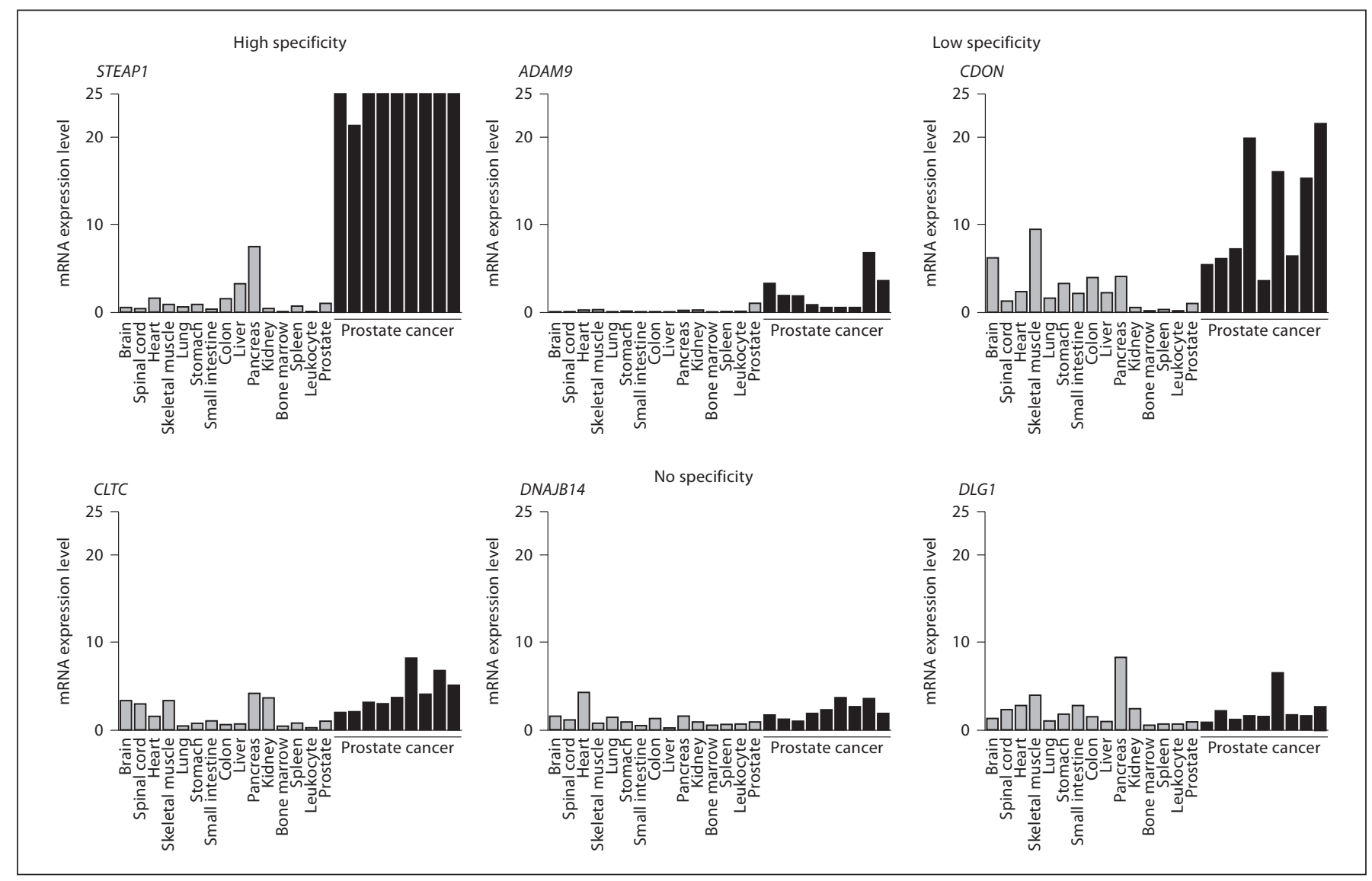

Fig. 1. Quantitative RT-PCR analysis of candidate genes that encode cell surface proteins in 15 normal tissues and 9 PCa samples. mRNA expression levels of STEAP1, ADAM9 and CDON were much higher in PCa samples than in normal tissues. In contrast, mRNA expression levels of CLTC, DNAJB14 and DLG1 were not significantly different between PCa and normal tissues.

ysis. Next, we examined the transition of CDO protein expression by Western blot analysis of cell extracts of DU145 transfected with CDON-specific siRNAs. Three types of siRNAs (siRNA1-3) were transfected into DU145. The expression of CDO protein in DU145 was substantially suppressed by treatment with siRNA1, siRNA2 and siRNA3 (fig. 2b). We used siRNA1 in the following experiments to knock down the endogenous $\mathrm{CDO}$ protein. We also performed a time course analysis (from days 2 to 8 ) of CDON expression after siRNA transfection in DU145 cells. We confirmed suppression of CDON mRNA expression from days 2 to 8 after siRNA1 transfection by quantitative RT-PCR (data not shown).

To investigate the possible antiproliferative effect of CDON knockdown, we performed an MTT assay 8 days after siRNA transfection (fig. 2c). DU145 cells were se- lected for high CDO protein expression. CDON siRNA1transfected DU145 cells showed significantly reduced viability relative to negative control siRNA-transfected DU145 cells. We performed the same assay using an LNCaP cell line that expressed low-level CDO protein. Reduced cell viability was not observed in siRNA1-transfected LNCaP cells compared with negative control siRNA-transfected LNCaP cells (fig. 2d). These results suggest that cell proliferation was activated or apoptosis was inhibited in CDON siRNA1-transfected DU145 cells. We examined the effect of CDON knockdown on the apoptotic susceptibility of DU145 cells to 5-FU. The frequency of apoptoitc cells in CDON siRNA1-transfected DU145 cells (mean, 71\%) was significantly higher than that in negative control siRNA-transfected DU145 cells (mean 42\%, $\mathrm{p}=0.0261$ ). 
Fig. 2. CDO protein expression and functional analysis. Western blot analysis of CDO protein in PCa cell lines (a). Western blot analysis of CDO protein in DU145 cells transfected with the negative control siRNA and CDON siRNA (siRNA1-3) (b). Effect of CDONknockdown on cell growth of DU145 cells (c). Cell growth was assessed by an MTT assay at 1,2, 4, and 8 days after seeding on 96-well plates in DU145 cells. Bars and error bars show means and SE of 3 different experiments. Effect of CDON knockdown on cell invasion of DU145 cells (d). After 1 and 2 days, invading cells were counted. Bars and error bars show means and SE of 3 different experiments. Cell growth was assessed by an MTT assay at 1, 2, 4 and 8 days after seeding on 96-well plates in LNCaP cells (e). Bars and error bars show means and SE of 3 different experiments. Effect of CDON knockdown on cell invasion of LNCaP cells (f). After 1 and 2 days, invading cells were counted. Bars and error bars show means and SE of 3 different experiments. NS $=$ Not significant. ${ }^{*} \mathrm{p}=0.0001 ;{ }^{* *} \mathrm{p}=$ 0.0229 .

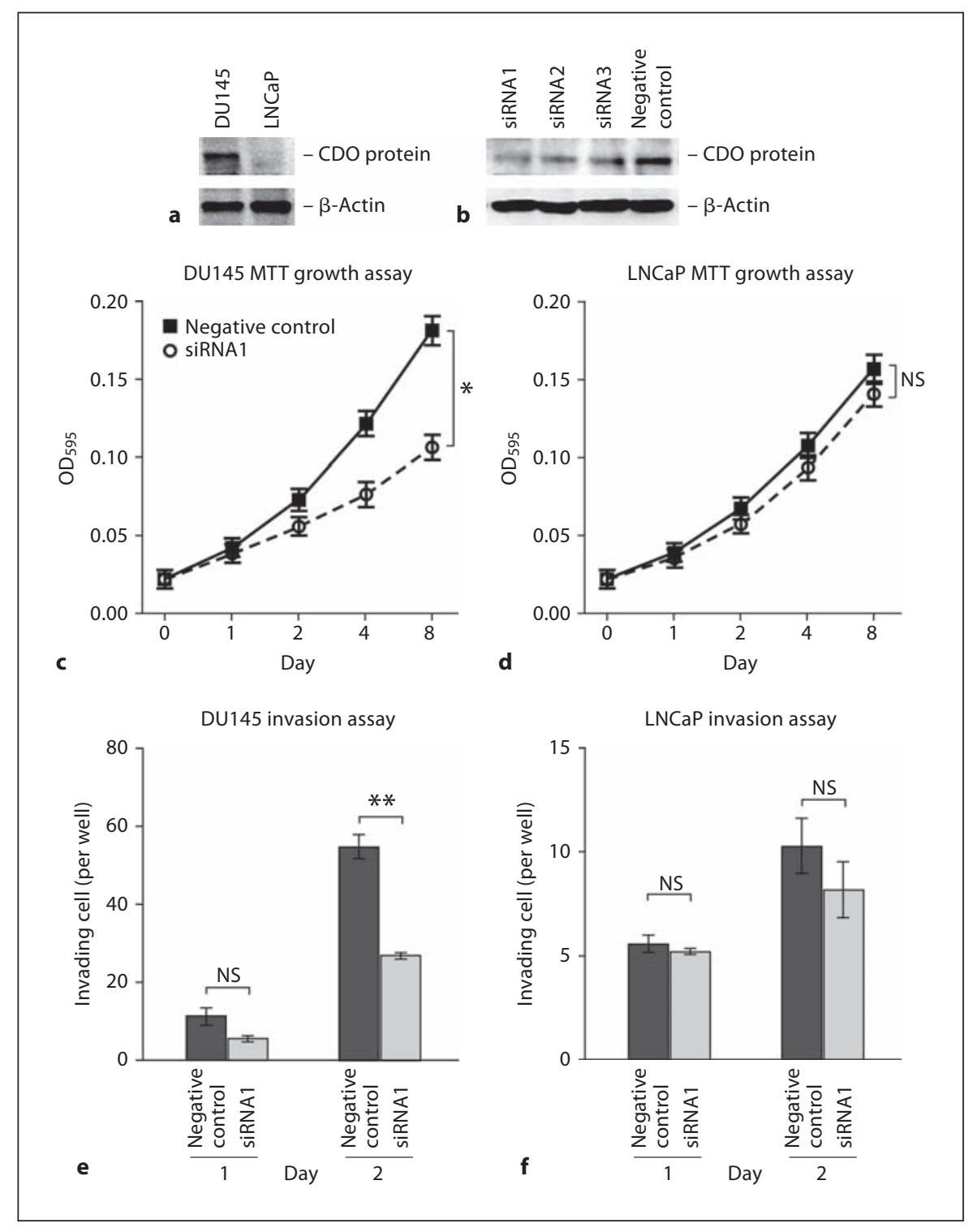

Next, to determine the possible role of $\mathrm{CDO}$ protein in the invasiveness of PCa cells, we used a transwell invasion assay (fig. 2e). On day 2, although cell viability was not significantly different between $C D O N$ knockdown DU145 cells and negative control siRNA-transfected DU145 cells, the invasiveness of CDON knockdown DU145 cells was 50\% less than that of the negative control siRNA-transfected DU145 cells. In contrast, invasion ability was not significantly different between CDON knockdown LNCaP cells and negative control siRNA-transfected LNCaP cells (fig. 2f). These results indicate that $\mathrm{CDO}$ protein stimulates invasion in $\mathrm{PCa}$ cells.

\section{Discussion}

We generated CAST libraries from 2 PCa cell lines, and identified several genes that encode transmembrane proteins present in PCa. Quantitative RT-PCR revealed that STEAP1, ADAM9 and CDON were expressed much more highly in PCa than in 15 types of normal tissues. STEAP1 showed the highest specificity for PCa. STEAP1 encodes the STEAP-1 protein, which is a six-transmembrane cell surface protein. It has been reported that mouse monoclonal antibodies specific to STEAP-1 extracellular loops inhibit the growth of prostate and bladder tumor xenografts [17]. ADAM9 showed the second highest spec- 
ificity for PCa. ADAM proteins are zinc metalloproteases, which are involved in ectodomain shedding of growth factors, adhesion molecules, cytokines and receptors. ADAM9 has been shown to have proteolytic activity and its substrates include pro-HB-EGF, pro-EGF, the FGF receptor and so on $[19,20]$. It has been reported that knockdown of ADAM9 results in increased apoptosis and increased sensitivity to radiation in PCa cells [21]. These results indicate that both STEAP-1 and ADAM9 are therapeutic targets for PCa. In contrast, little is known about CDON expression in human cancers. In the present study, quantitative RT-PCR revealed that CDON was overexpressed in $83 \%$ of the PCa tissue samples, and knockdown of CDON in PCa cells induced 5-FU-induced apoptosis and inhibited invasion ability. These results suggest that $C D O N$ has the potential to constitute a therapeutic target for PCa.

The biological function of CDO protein is poorly understood. Previously, Kang et al. reported that CDO protein may have transformation suppressor function [10]. Expression of CDO protein was down-regulated in a rat embryo fibroblast cell line that was transformed by various oncogenes [10]. In addition, CDO protein levels were reduced when confluent cells were stimulated to re-enter the cell cycle. These results suggest that $\mathrm{CDO}$ protein may serve as a negative regulator of cell proliferation, transformation, and/or tumorigenesis. In the present study, knockdown of CDON in PCa cells induced 5-FU-induced apoptosis and inhibited invasion ability. Our present results are inconsistent with the previous reports, in which the function of CDO protein was investigated in rat embryo fibroblasts [10]. Here, we found that CDO protein in PCa cells may be different from fibroblasts.

On the other hand, it has been shown that the Sonic Hedgehog (SHh) directory interacts with CDO protein [22], and CDO protein functions to positively regulate SHh signaling in vivo and in vitro [23]. The Hh signaling pathway plays a central role during embryonic development and acts in stem cell renewal as well as tissue repair
[24]. Hh ligands act via several components, including Patched and Smoothened transmembrane receptors, and lead to the activation of the Gli, a zinc-finger transcription factor. Several lines of evidence have indicated an association between Hh pathway activation and initiation or growth of several human cancers, including basal cell carcinomas, medulloblastomas and PCa $[25,26]$. Therefore, it is possible that in addition to Patched, CDO protein also leads to the activation of the SHh pathway and participates in tumor cell growth in PCa.

Although CDON mRNA upregulation was observed in bulk PCa tissues by quantitative RT-PCR, expression and distribution of CDO protein in PCa tissues remains unclear. In this viewpoint, we performed immunohistochemical analysis in PCa tissue samples; however, obvious membranous staining was not found. The antibody against CDO protein used in the present study is not suitable for immunostaining because the antibody against CDO protein detected multiple bands on Western blots. Production of a specific antibody against CDO protein for immunostaining is required.

In summary, our present study yielded a list of genes that encode transmembrane proteins present in PCa. Our current data also provide information with respect to the expression of these genes throughout the body. We found that $C D O N$ is overexpressed in $\mathrm{PCa}$, and $C D O N$ expression is narrowly restricted in normal tissues. Functional and immunohistochemical analysis will certify whether CDON may constitute a therapeutic target for PCa.

\section{Acknowledgements}

We thank Mr. Shinichi Norimura for his excellent technical assistance and advice. This work was carried out with the kind cooperation of the Research Center for Molecular Medicine, Faculty of Medicine, Hiroshima University. We thank the Analysis Center of Life Science, Hiroshima University, for the use of their facilities. This work was supported in part by Grants-in-Aid for Cancer Research from the Ministry of Education, Culture, Science, Sports and Technology of Japan.

\section{References}

1 Baade PD, Youlden DR, Krnjacki LJ: International epidemiology of prostate cancer: geographical distribution and secular trends. Mol Nutr Food Res 2009;53:171-184.

$\checkmark 2$ Carter HB: Prostate cancers in men with low PSA levels - must we find them? N Engl J Med 2004;350:2292-2294.

CAST Analysis of Prostate Cancer
3 Chang SS, Kibel AS: The role of systemic cytotoxic therapy for prostate cancer. BJU Int 2009;103:8-17.

4 Isaacs W, De Marzo A, Nelson WG: Focus on prostate cancer. Cancer Cell 2002;2:113-116.

5 Buckhaults P, Rago C, St Croix B, et al: Secreted and cell surface genes expressed in benign and malignant colorectal tumors. Cancer Res 2001;61:6996-7001.
-6 Ferguson DA, Muenster MR, Zang Q, et al: Selective identification of secreted and transmembrane breast cancer markers using Escherichia coli ampicillin secretion trap. Cancer Res 2005;65:8209-8217.

7 von Heijne G: A new method for predicting signal sequence cleavage sites. Nucleic Acids Res 1986;14:4683-4690. 
8 Kadonaga JT, Gautier AE, Straus DR, Charles AD, Edge MD, Knowles JR: The role of the $\beta$-lactamase signal sequence in the secretion of proteins by Escherichia coli. J Biol Chem 1984;259:2149-2154.

-9 Anami K, Oue N, Noguchi T et al: Search for transmembrane protein in gastric cancer by the Escherichia coli ampicillin secretion trap: expression of DSC2 in gastric cancer with intestinal phenotype. J Pathol 2010;221:275284.

-10 Kang JS, Gao M, Feinleib JL, Cotter PD, Guadagno SN, Krauss RS: CDO: an oncogene-, serum-, and anchorage-regulated member of the Ig/fibronectin type III repeat family. J Cell Biol 1997;138:203-213.

-11 Kang JS, Mulieri PJ, Hu Y, Taliana L, Krauss RS: BOC, an Ig superfamily member, associates with $\mathrm{CDO}$ to positively regulate myogenic differentiation. EMBO J 2002;21:114124.

-12 Gibson UE, Heid CA, Williams PM: A novel method for real time quantitative RT-PCR. Genome Res 1996;6:995-1001.

13 Kondo T, Oue N, Yoshida K, et al: Expression of POT1 is associated with tumor stage and telomere length in gastric carcinoma. Cancer Res 2004;64:523-529.
14 Yasui W, Sano T, Nishimura K, et al: Expression of $\mathrm{P}$-cadherin in gastric carcinomas and its reduction in tumor progression. Int $\mathrm{J}$ Cancer 1993;54:49-52.

15 Alley MC, Scudiero DA, Monks A, et al: Feasibility of drug screening with panels of human tumor cell lines using a microculture tetrazolium assay. Cancer Res 1988;48:589601.

16 Sakamoto N, Oue N, Noguchi T, et al: Serial analysis of gene expression of esophageal squamous cell carcinoma: ADAMTS16 is upregulated in esophageal squamous cell carcinoma. Cancer Sci 2010;101:1038-1044.

17 Challita-Eid PM, Morrison K, Etessami S, et al: Monoclonal antibodies to six-transmembrane epithelial antigen of the prostate- 1 inhibit intercellular communication in vitro and growth of human tumor xenografts in vivo. Cancer Res 2007;67:5798-5805.

18 Fritzsche FR, Jung M, Tölle A, et al: ADAM9 expression is a significant and independent prognostic marker of PSA relapse in prostate cancer. Eur Urol 2008;54:1097-1106.

19 Izumi Y, Hirata M, Hasuwa H, et al: A metalloprotease-disintegrin, MDC9/meltrin- $\gamma /$ ADAM9 and PKC $\delta$ are involved in TPA-induced ectodomain shedding of membraneanchored heparin-binding EGF-like growth factor. EMBO J 1998;17:7260-7272.
20 Peduto L, Reuter VE, Shaffer DR, Scher HI, Blobel CP: Critical function for ADAM9 in mouse prostate cancer. Cancer Res 2005;65: 9312-9319.

21 Josson S, Anderson CS, Sung SY, et al: Inhibition of ADAM9 expression induces epithelial phenotypic alterations and sensitizes human prostate cancer cells to radiation and chemotherapy. Prostate 2011;71:232-240.

22 McLellan JS, Zheng X, Hauk G, Ghirlando R, Beachy PA, Leahy DJ: The mode of Hedgehog binding to Ihog homologues is not conserved across different phyla. Nature 2008;455:979983.

23 Zhang W, Kang JS, Cole F, Yi MJ, Krauss RS: CDO functions at multiple points in the Sonic Hedgehog pathway, and CDO-deficient mice accurately model human holoprosencephaly. Dev Cell 2006;10:657-665.

-24 Pasca di Magliano M, Hebrok M: Hedgehog signalling in cancer formation and maintenance. Nat Rev Cancer 2003;3:903-911.

$\checkmark 25$ Bale AE: Hedgehog signaling and human disease. Annu Rev Genomics Hum Genet 2002;3:47-65.

26 Sanchez P, Hernández AM, Stecca B, et al: Inhibition of prostate cancer proliferation by interference with SONIC HEDGEHOGGLI1 signaling. Proc Natl Acad Sci USA 2004;101:12561-12566 